\title{
Kommunikation mit einem Demenzkranken
}

\section{Patienteninformation}

Stellen Sie sich vor, Sie haben ein sehr behagliches Gefühl, weil Ihre Tochter zu Besuch ist. Sie sprechen über ihren gemeinsamen Ausflug neulich - Sie wissen nicht mehr viel darüber, aber sie hat Fotos mitgebracht. Auf einem Foto erkennen Sie erfreut das Café wieder. Der Mann neben ihnen sei ein Bekannter, der Ihnen beste Grüße ausrichte, so Ihre Tochter. Die Süßspeise war wie immer besonders gut dort - Ihnen fällt die Bezeichnung dafür gerade nicht ein, aber Ihre Umschreibung mit viel Gestikulieren wird dank einer Rückfrage der Tochter bald verstanden. Sie erzählen ihr daraufhin von einem lange zurück liegenden Ereignis, das vage mit dem Café assoziiert ist. Ihre Tochter schaut unverständig, lässt Ihnen aber Zeit. Sie wissen für einige Momente nicht weiter und haben fast vergessen worum es überhaupt ging, werden unzufrieden, Ihre Gedanken schweifen ab. Ihre Tochter berührt Ihren Arm, lenkt Ihre Aufmerksamkeit zurück auf das Foto und sie beide sind sich einig, dass das Wetter fantastisch war.

\section{Gestörte Sprache und Kommuni- kation bei Demenz \\ $\nabla$}

In einer derartigen, eher gelungenen Kommunikationssituation könnten Sie sich als Demenzkranker mit einem recht gut informierten Gesprächspartner befinden. Bereits im frühen Stadium einer degenerativen Demenzerkrankung treten häufig Wortfindungsstörungen auf, die die Kommunikation beeinträchtigen können. Darüber hinaus ist das Störungsbild nicht einheitlich und verändert sich im Krankheitsverlauf. Die Kommunikation kann, wie bei der Alzheimer-Demenz, durch das Nachlassen von Gedächtnis und Aufmerksamkeit erschwert sein. Dabei bleiben Persönlichkeit, Gefühlsebene, Körpersprache und Gesprächsregeln meist erhalten. Sprachliche Leistungen wie das Formulieren und Verstehen von wenig konkreten Wörtern und komplexen Sätzen können zunehmend betroffen sein.
Trotz deutlicher Sprachstörungen können aber, je nach Demenzform, Denken und Erinnern gut erhalten bleiben.

\section{Anpassung an den betroffenen Gesprächspartner}

Für eine gelungene Kommunikation kann der gesunde Gesprächspartner die sprachlich-kognitiven Stärken und Schwächen des Demenzkranken einzuschätzen versuchen. Er/sie sollte sich möglichst einfühlsam verhalten, störende Einflüsse reduzieren und Verantwortung für das Gespräch übernehmen, indem er/sie ...

- die Defizite des Gesprächspartners auszugleichen sucht,

- die bestehenden Fähigkeiten erkennt und würdigt,

- die Gefühlsebene anspricht und eine angenehme Atmosphäre schafft,

- die inhaltliche Verständigung wichtiger nimmt als die sprachlich-formale Übermittlung und

- die Äußerungen des Betroffenen weder zu wörtlich noch zu persönlich nimmt.

\section{„Faustregeln“ für die Kommuni-} kation

- Gute Stimmung, Berührung, Wärme, Intonation, eine freundliche Stimme werden trotz sprachlich-kognitiver Defizite wahrgenommen. Bei den meisten Demenzformen sind die Gefühle der Betroffenen adäquat und können zumindest nicht-sprachlich ausgedrückt werden.

- Bei Personen mit Gedächtnisdefiziten sind frühe Erlebnisse am besten erhalten, sodass lebensgeschichtliche Erinnerungen ein beliebtes Thema sind.

- Bilder, Fotos und Musik können die Unterhaltung anregen. Zeichnen, Körpersprache, Singen und gegebenenfalls Schrift können den Ausdruck fördern.

- Bei Schwächen der Merkfähigkeit sowie bei kurzen oder ungrammatischen Sätzen sollten Sie sich darauf einstel- len, auch ihre eigenen Sätze und die enthaltenen Sachverhalte nicht zu komplex anzulegen.

- Bei Verlust des roten Fadens hilft das Wiederholen zuletzt gesprochener Worte oder des aktuellen Themas. Zudem können Hilfsmittel und Situationen, die an Kommunikationsziele erinnern (Foto, Bild, Schrift, Umgebung), helfen.

- Bei konfus anmutenden Informationen, häufiger Wiederholung des Gesagten oder schnellem Vergessen der Gesprächsinhalte ist für eine Verständigung entscheidend, dass Sie die Gesprächsführung übernehmen.

- Bei Wortfindungsschwierigkeiten ausreichend Zeit lassen, Vorschläge bieten oder nicht-sprachliche Kommunikation anregen. Bei Verwechslungen von Lauten oder Wörtern können Zusammenhänge verwirrend bleiben. Durch rückversichernde Fragen und Rückmeldungen kann die Verständigung sicher gestellt werden („Meinst Du Tiramisu? Nein? Donauwellen? Ah ja!“).

- Falls die Verständigung trotz Bemühungen nicht klappt, können Sie schon um der guten Stimmung willen - auch mal lächelnd aufgeben, einen Themenwechsel einleiten oder auf einen späteren Zeitpunkt verweisen.

- Die betroffenen Personen verstehen mehr als sie sprechen können. Achten Sie auf Hinweise für schnelle Ermüdung und gedankliche Verlangsamung beim Gesprächspartner sowie darauf, sich eindeutig und konkret auszudrücken.

Priv.-Doz. Dr. Stefanie Abel, Aachen,

überreicht durch Thieme und 